\title{
Campaña influenza 2020 en contexto de pandemia por SARS-CoV-2: una experiencia inédita de salud pública en Chile
}

\section{Seasonal influenza campaign 2020 in the context of SARS-CoV-2 pandemic: an unprecedented public health experience in Chile}

\author{
Magdalena Bastías', Marcela Avendaño', Felipe Muñoz', Iván Brstilo', Andrés Álvarez', Pamela Burgos', Daniela Espinoza', \\ Marcia Núñez ${ }^{1}$, Juan Rodríguez, Marcela Rivas' ${ }^{1}$ Rosa Solar', Leslie Soto ${ }^{1}$ y Cecilia González ${ }^{1}$
}

'Departamento de Inmunizaciones, Ministerio de Salud de Chile.

Recibido: 10 de marzo de 2021

\section{Resumen}

Desde 1982, cada año el Departamento de Inmunizaciones del Ministerio de Salud de Chile lleva a cabo la campaña de vacunación contra influenza junto con las Secretarías Regionales MinisterialesSEREMI, Servicios de Salud y centros de atención primaria de salud. Con los objetivos de prevenir mortalidad y morbilidad grave en grupos de mayor riesgo y de preservar la integridad de los servicios de salud, hasta el 2020 las campañas de vacunación contra influenza serían las más grandes implementadas en Chile, para dar paso, el 2021, a la vacunación contra SARS-CoV-2. Obedeciendo a cambios demográficos y epidemiológicos locales y acogiendo los avances científicos sobre seguridad e inmunogenicidad de la vacuna, el incremento de las vacunas influenza disponibles en Chile forma parte de la planificación anual de la campaña. El 2020, sin embargo, la Campaña Influenza tuvo que ser re-planificada en curso como consecuencia de la incorporación de nuevos grupos a vacunar según dispuso la modificación de la alerta sanitaria por brote de SARS-CoV-2 del 6 de marzo de 2020. Así, de 6.799.800 de dosis, el Departamento de Inmunizaciones logró en menos de dos meses aumentar la disponibilidad a 8.480.325, y cumplir con el compromiso de garantizar el acceso de los grupos de riesgo al beneficio de la vacunación estatal gratuita.

Palabras clave: vacunación anti-influenza; programa de inmunización; vacunación masiva; campaña de vacunación; cobertura de vacunación; influenza humana; pandemia; SARS-CoV-2; Chile.

\section{Abstract}

In Chile, the Immunization Department of the Ministry of Health has carried out the seasonal influenza vaccination campaign annually since 1982 in collaboration with the national health services, regional health offices, and primary health care centres. With the aim of preventing deaths and serious morbidity in high-risk groups and preserving the integrity of health services, the seasonal influenza campaign had been the largest implemented in Chile until 2020, since in 2021 the vaccination campaign against SARS-CoV-2 is expected to become the largest ever implemented. In response to local demographic and epidemiological changes, and taking into account the new scientific evidence on the safety and immunogenicity of vaccines, the influenza vaccines available in Chile would increase annually as a result of campaign planning. In 2020, the influenza campaign had to be re-planned while in progress due to the addition of new high-risk groups to be vaccinated in accordance with the SARS-CoV-2 pandemic health alert modification of March 6th, 2020. Over the course of three weeks, the Immunization Department managed to increase the doses of available influenza vaccines from $6,799,800$ previously agreed upon to $8,480,325$ and thus serve high-risk groups, guaranteeing their access to state funded influenza vaccination.

Keywords: influenza vaccination; immunization programs; mass vaccination; vaccination campaign; vaccine coverage; human influenza, SARS-CoV-2, pandemic, Chile. 


\section{Introducción}

a entrega de servicios de inmunización programática por parte del Departamento de Inmunizaciones (DI) del Ministerio de Salud (MINSAL) de Chile busca brindar protección a la población residente en el país frente a enfermedades prevenibles por vacunas ${ }^{1}$. Desde 1982, cada año el DI lleva a cabo la Campaña Influenza junto con las Secretarías Regionales Ministeriales -SEREMI, Servicios de Salud a nivel medio y centros de atención primaria de salud a nivel ejecutor ${ }^{2}$, una campaña de vacunación poblacional contra la influenza estacional que comienza el mes de marzo y termina cuando se ha alcanzado la meta de cobertura $(\geq 85 \%)$, soliendo extenderse hasta el mes de diciembre. Sus objetivos son la prevención de mortalidad y morbilidad grave en subgrupos de la población definidos por las condiciones biomédicas que se asocian a mayor riesgo de muerte y complicaciones causadas por la infección por el virus influenza y la preservación de la integridad de los servicios de salud ${ }^{3}$.

Obedeciendo a cambios demográficos y epidemiológicos locales y acogiendo los avances científicos sobre seguridad e inmunogenicidad de la vacuna contra influenza, la cantidad de vacunas influenza proporcionada por el MINSAL a la población se incrementa anualmente. También, frente a situaciones extraordinarias como las necesidades sanitarias secundarias a desastres naturales, el DI ha procurado aumentar la disponibilidad de vacuna influenza para responder a esas demandas específicas. Siguiendo recomendaciones de la Organización Mundial de la Salud, en un comienzo, los grupos objetivo de vacunar contra influenza fueron las personas mayores de 65 años, personas que padecen enfermedad crónica y personal de salud, lo que llevó a una adquisición de 2.300.000 vacunas en el período entre 1982 y 2003. El año 2004 se incorporó a las mujeres embarazadas a partir de la semana 14 de gestación, lo que significó 250.000 dosis adicionales cada año, aproximadamente. El 2006, se sumaron los lactantes sanos y el 2015, niños entre 3 y 5 años, los que, en adición a los otros grupos de riesgo definidos previamente, significaron para el año 2019 una adquisición de 5.677.200 de vacunas influenza. El 2020, la vacunación de las mujeres embarazadas se amplió a cualquier momento de la gestación y la de niños, hasta los 10 años o $5^{\circ}$ básico de enseñanza escolar, lo que, sumado a los otros grupos de riesgo, se tradujo en una adquisición de 6.799.800 de vacunas influenza para el 2020, inicialmente.

El 30 de enero de 2020, la Organización Mundial de la Salud (OMS) declaró al brote de COVID-19 una emergencia de salud pública de importancia internacional (ESPII), y el 11 de marzo 2020, una pandemia ${ }^{4,5}$. En Chile, el decreto $\mathrm{N}^{\mathrm{o}} 4$ del 5 de enero de 2020 declaró alerta sanitaria por un año y otorgó facultades extraordinarias a las Subsecretarías de Salud Pública y Redes Asistenciales, SEREMI, Servicios de Salud, Central Nacional de Abastecimiento-CENABAST, Superintendencia de Salud, Fondo Nacional de Salud-FONASA y al Instituto de Salud Pública por ESPII debido al brote del nuevo coronavirus (2019-NCOV- hoy en día SARS-CoV-2) El 6 de marzo de 2020 se modificó el decreto $\mathrm{N}^{\mathrm{o}} 4$ y, entre los cambios, se agregó el numeral 21 al artículo $3^{\circ}$, señalando: "Disponer la vacunación de personas que no se encuentren dentro de los grupos objetivos definidos previamente por decreto del Ministro de Salud."7.

Siguiendo la indicación de la Subsecretaría de Salud Pública, basada en la recomendación del Comité de Operaciones de Emergencias convocado por el Ministro del Interior el 16 de marzo, el DI comenzó a ejecutar la entrega de vacunas influenza a nuevos grupos dentro de la categoría Otras prioridades de la Campaña Influenza 2020, en paralelo al llamado que hacía la autoridad sanitaria a vacunarse oportunamente contra influenza para evitar la co-existencia del virus influenza con SARS$\mathrm{CoV}-2^{8}$. En este artículo, describimos qué significó para el Departamento de Inmunizaciones de Chile la vacunación de nuevos grupos en la Campaña Influenza 2020 y cómo esta se desarrolló en comparación con años anteriores.

\section{Objetivo}

Describir la experiencia del Departamento de Inmunizaciones en la replanificación e implementación de la Campaña Influenza 2020 en el contexto de pandemia por SARS-CoV-2.

\section{Material y Métodos}

Descripción de una experiencia de salud pública en el control de una enfermedad inmunoprevenible, la influenza, en contexto de pandemia por SARS-CoV-2. Se recopilaron y revisaron antecedentes técnicos, normativos, legales e informativos relacionados a la Campaña Influenza 2020.

\section{Resultados}

\section{Nuevos grupos de vacunación y adquisición de dosis}

De acuerdo a la planificación de la Campaña Influenza 2020, que comenzó en agosto 2019, los siguientes fueron los grupos objetivo de vacunar, inicialmente ${ }^{3,9}$ :

- Trabajadores, voluntarios, estudiantes que se desempeñaran en servicios asistenciales o instituciones de salud públicas y privadas.

- Mujeres embarazadas, en cualquier semana de gestación. 
- Niños desde los 6 meses de edad y hasta los 10 años o $5^{\circ}$ año básico de enseñanza escolar.

- Personas de 65 años y más.

- Trabajadores de avícolas y de criaderos de cerdos.

- Personas entre 11 y 64 años de edad que padecieran alguna de las siguientes condiciones de riesgo:

- Enfermedad pulmonar crónica: asma bronquial, EPOC, fibrosis quística, fibrosis pulmonar de cualquier causa.

- Enfermedad neurológica: neuromuscular congénita o adquirida, que determine trastornos de la deglución o del manejo de secreciones respiratorias, epilepsia refractaria a tratamiento.

- Enfermedad renal crónica: insuficiencia renal en etapa 4 o mayor, en diálisis.

- Enfermedad hepática crónica: cirrosis, hepatitis crónica, hepatopatías.

- Enfermedades metabólicas: diabetes mellitus, enfermedades congénitas del metabolismo.

- Cardiopatía: congénita, reumática, isquémica y miocardiopatía de cualquier causa.

- Hipertensión arterial en tratamiento farmacológico.

- Obesidad: IMC $\geq 30$.

- Enfermedad autoinmune: lupus sistémico, escleroderma, artritis reumatoide, enfermedad de Crohn y otras.

- Cáncer en tratamiento con radioterapia, quimioterapia, terapias hormonales o medidas paliativas de cualquier tipo.

- Inmunodeficiencias: congénitas o adquiridas.

- Otras prioridades:

- Establecimientos de atención cerrada (Servicio Nacional de Menores-SENAME, establecimientos psiquiátricos) y personal de apoyo que desempeñe funciones en estos establecimientos.

- Población privada de libertad.

- Gendarmería.

- Personal que trabaja en jardines infantiles.

- Profesores de kínder a $5^{\circ}$ año básico de enseñanza escolar.

- Cuidadores y contactos intra-domiciliarios de personas postradas.

- Estrategia capullo para prematuros extremos $(<\mathrm{de}$ 32 semanas o 1.500 gramos al nacer) y prematuros con patologías definidas en Recomendaciones para la vacunación de pacientes con necesidades especiales por patología o situaciones de riesgo ${ }^{10}$.

- Alumnos de escuelas de formación en Fuerzas Armadas (Ejército-Armada-Fuerza Aérea) y en Fuerza de Orden y Seguridad Pública (Carabineros- Policía de Investigaciones).

- Carabineros que trabajan en terreno.

- Personas en situación de calle.

- Trabajadores sexuales.
- Bomberos.

- Funcionarios públicos (solicitud de jefatura de la institución y aprobación del Departamento de Inmunizaciones y SEREMI, según corresponda).

- Personas residentes en zonas extremas ( $\sin$ acceso a un centro de salud).

Definidos los grupos objetivo de vacunar durante la campaña, la estimación de la población a vacunar fue de 6.799 .800 personas, alrededor de $39 \%$ de la población total país, según las proyecciones para el año $2020^{9}$.

Siguiendo la recomendación de la OMS para vacunación en el Hemisferio Sur $2020^{11}$, la vacuna adquirida por el DI para la Campaña Influenza 2020 fue Influvac ${ }^{\circledR}$, Reg. ISP: B-1007/15, vacuna inactivada trivalente, cepas A/ Brisbane/02/2018 (H1N1) pdm09 derivada de A/Brisbane/02/2018, IVR-190, A/South Australia/34/2019(H3N2) derivada de A/South Australia/34/2019, IVR-197 y B/ Washington/02/2019 derivada de B/Washington/02/2019 salvaje. La licitación de la compra se hizo en octubre de 2019 y alcanzó un costo de $\$ 16.469 .015 .472$, equivalente a 23\% del presupuesto del DI para el año 2020.

La primera distribución a los depósitos de vacunas e inmunoglobulinas (DVI) se hizo la última semana de febrero 2020. Para su ejecución, la campaña contó con estrategias de refuerzo de vacunación que incluyeron la contratación de equipos vacunadores, de técnicos en enfermería nivel superior y de digitadores por parte de SEREMI, además del traspaso de recursos desde el nivel central a SEREMI y del refuerzo de la seguridad en los DVI regionales.

A raíz de la modificación del Decreto $N^{\circ} 4$ de 2020 del MINSAL que declaró la alerta sanitaria por la emergencia de salud pública de importancia internacional por el brote de SARS-CoV $-2^{7}$ y por orden de la Subsecretaría de Salud Pública, en marzo de 2020 el DI incluyó nuevos grupos dentro de la categoría Otras prioridades de la campaña. De esta manera, el DI comenzó la re-planificación de la Campaña Influenza 2020 que, luego de un análisis sobre el riesgo de exposición simultánea a Influenza y SARS$\mathrm{CoV}-2$, que fuera mayor al del resto de la población, incorporó a los siguientes nuevos grupos:

- Ejército de Chile, Fuerza Aérea de Chile, Armada de Chile, y Subsecretaría de Defensa, Subsecretaría para las Fuerzas Armadas y Estado Mayor Conjunto.

- Transportistas: conductores del transporte público, buses, camiones, portuarios, personal en filiales EFE y Metro.

- Ministerio de Obras Públicas y Superintendencia de Servicios Sanitarios.

- Ministerio de Energía.

- Superintendencia de Electricidad y Combustible.

- Carabineros de Chile.

- Policía de Investigaciones. 
- Recolectores de basura.

- ONEMI.

- Corporación Nacional Forestal.

La definición de los nuevos grupos a vacunar contra influenza se tradujo en la necesidad de, a su vez, adquirir nuevas dosis de vacuna. Para ello, el 17 de marzo de 2020 el DI inició las gestiones para la compra de vacunas influenza por distintos medios: extensión de contrato con laboratorio que le proveyera la primera remesa para la campaña, licitación y compra a través del Fondo Rotatorio de la Organización Panamericana de la Salud. Así, el 8 de mayo de 2020 se concretó la última compra de vacunas para completar las 1.680 .525 dosis necesarias para cubrir a los nuevos grupos, lo que significó un costo total de \$6.445.536.867. En consecuencia, la Campaña Influenza 2020 adquirió un total de 8.480 .325 dosis de vacuna, como suma de la estimación inicial de población beneficiaria y los nuevos grupos incorporados como parte de las medidas extraordinarias que impuso la alerta sanitaria por pandemia de SARS-CoV-2.

\section{Lineamientos técnicos y operativos}

Las dosis de vacuna influenza adquiridas por la incorporación de nuevos grupos, su distribución y administración, al igual que las remesas de la primera licitación, quedaron bajo los Lineamientos Técnicos Operativos Vacuna Anti-influenza 2020, publicados y comunicados el 18 de marzo de 2020 que, a su vez, contemplan la Norma Técnica $N^{\circ} 147 / 2013$ Buenas prácticas de almacenamiento y distribución, la Norma General Técnica No973/2012 sobre Procedimientos Operativos Estandarizados para Asegurar la Calidad en la Ejecución del Programa Nacional de Inmunizaciones y la Guía de Vacunación Segura ${ }^{12}$. Estos lineamientos entregaron las direcciones de acción a los SEREMI, nivel medio y ejecutor en relación a la vacunación contra influenza 2020, incluyendo los aspectos de comunicación entre niveles y con las Autoridades Municipales para la definición de estrategias para el cumplimiento de los objetivos sanitarios, estándares técnicos y plazos definidos por el MINSAL para ejecución de la campaña, el plan de comunicación social, conformación de los equipos de trabajo, entrenamiento técnico y supervisión de la ejecución, el retiro y recepción de vacunas desde un DVI, cumplimiento de la cadena de frío, identificación de poblaciones beneficiarias y planificación de las distintas modalidades de vacunación (grupos cautivos, intramural, domiciliaria, población en tránsito y sitios de alta concentración), administración y cuidados de la vacunación, monitorización, notificación y acciones frente a eventos supuestamente atribuidos a la vacunación o inmunización (ESAVI) y de errores programáticos (EPRO), ingreso de información al Registro Nacional de Inmunizaciones, consejería ante el rechazo a la vacunación y registro del evento, seguimiento y verificación de coberturas de vacunación, la evaluación de la campaña y el informe regional.

\section{Vacunación contra influenza 2020 respecto de años anteriores}

La Campaña Influenza 2020, que se implementó bajo el marco de vacunación segura en tiempos de pandemia que impuso medidas de prevención de infección y diseminación de SARS-CoV-2, tanto para los equipos de salud como para la población beneficiaria ${ }^{13-16}$, comenzó el 16 de marzo y los últimos vacunados se registraron el 10 de octubre de 2020.

El primer día hábil de campaña se administraron 736.481 y 799.964 el segundo día hábil, lo que significó entre 3,3 a 46,3 y entre 3,4 a 16,8 veces más que lo registrado los días hábiles $2^{\circ}$ y $3^{\circ}$, respectivamente, de los siete años previos (Tabla 1). Las 799.964 dosis del segundo día hábil de campaña constituyeron, además, el valor máximo de dosis de vacuna contra influenza alcanzado el 2020, cifra que fue entre 3,4 y 10,3 veces más que los valores máximos registrados los años anteriores (Tabla 1).

\begin{tabular}{|c|c|c|c|c|c|c|c|c|}
\hline & 2013 & 2014 & 2015 & 2016 & 2017 & 2018 & 2019 & 2020 \\
\hline Día hábil 1 & 63.910 & 78.533 & 15.895 & 50.741 & 69.831 & 221.033 & 80.397 & 736.481 \\
\hline Día hábil 2 & 95.153 & 98.245 & 47.631 & 69.215 & 76.394 & 232.402 & 87.143 & 799.964 \\
\hline Día hábil 3 & 91.928 & 101.772 & 60.874 & 80.816 & 75.959 & 203.642 & 98.321 & 715.583 \\
\hline Día hábil 4 & 99.235 & 103.960 & 70.629 & 80.622 & 112.238 & 219.143 & 131.984 & 604.814 \\
\hline Día hábil 5 & 88.837 & 93.368 & 97.607 & 76.100 & 113.996 & 186.717 & 129.176 & 461.576 \\
\hline $\begin{array}{l}\text { Máximo diario y día de campaña } \\
\text { alcanzado }\end{array}$ & $\begin{array}{l}77.612 \\
\text { día } 16\end{array}$ & $\begin{array}{c}108.138 \\
\text { día } 9\end{array}$ & $\begin{array}{c}129.836 \\
\text { día } 17\end{array}$ & $\begin{array}{l}105.187 \\
\text { día } 10\end{array}$ & $\begin{array}{l}128.643 \\
\text { día } 13\end{array}$ & $\begin{array}{l}232.402 \\
\text { día } 2\end{array}$ & $\begin{array}{l}138.190 \\
\text { día } 13\end{array}$ & $\begin{array}{c}799.964 \\
\text { día } 2\end{array}$ \\
\hline
\end{tabular}


En comparación al 2018 y 2019, la mayor demanda por vacuna influenza que se experimentó el 2020 se extendió hasta el 27 de marzo, día después del cual ésta se ubicó por debajo de lo observado en la misma fecha en años anteriores (Figura 1); sin embargo, el porcentaje de avance de vacunación 2020 ya alcanzaba el $67 \%$, mientras que a la misma fecha era de $24 \%$ el 2019 y $50 \%$ el 2018 .

La velocidad del avance de la demanda de vacuna influenza el 2020 fue superior respecto de años anteriores $\mathrm{y}$, tomando los últimos siete años como comparación, el valor máximo diario de vacunación ocurrió entre 7 y 15 días más rápido que años previos, a excepción del año 2018 en que también fue en el segundo día de campaña (Tabla 1). La mayor velocidad de avance de la campaña 2020 (Figura 2) significó, además, alrededor de seis semanas de ventaja respecto del 2019 y 2018 en alcanzar
Figura 1. Evolución de la vacunación diaria en las campañas de vacunación contra influenza en Chile, años 2018,2019 y 2020
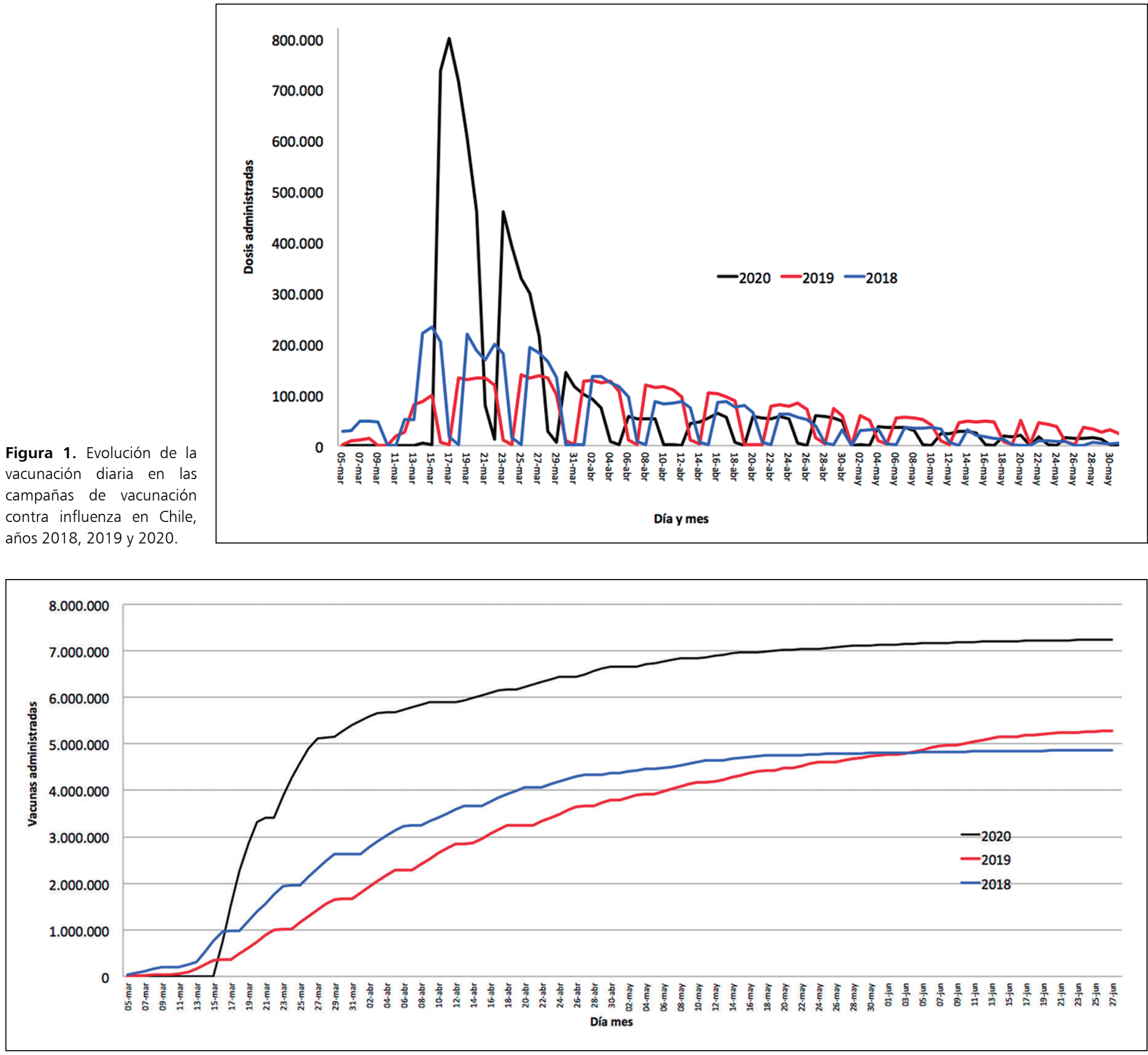

Figura 2. Avance acumulado de la vacunación contra Influenza en Chile, años 2018, 2019 y 2020. 
la meta de cobertura $85 \%$ (esquema de vacunación completo), ocurriendo esto el 27 de abril en el año 2020, y el 13 y 11 de junio el 2019 y 2018 , respectivamente. Al 31 de diciembre de 2020, la cobertura de vacunación contra influenza en el país fue $98,7 \%$.

En relación a los eventos supuestamente atribuidos a la vacunación o inmunización, ESAVI, la tasa de notificación el 2020 fue 1,7 ESAVI por 100.000 dosis de vacuna influenza administrada, en tanto el 2019 fue 3,8/100.000 y el 2018, 3,9/100.000*.

\section{Discusión y Conclusiones}

A raíz de la situación sanitaria por pandemia de SARSCoV-2 y de la alerta sanitaria correspondiente que se decretó en Chile, el DI se vio frente a la necesidad de replanificar la Campaña Influenza 2020 que ya se encontraba en curso, tanto por la incorporación de nuevos grupos a vacunar por su mayor riesgo de exposición a influenza y a SARS-CoV-2, como por la compra y gestión de las nuevas dosis de vacuna.

La definición de los nuevos grupos a vacunar contra influenza significó una población adicional a lo planificado para la campaña, por lo que fue necesario adquirir, extraordinariamente, 1.680 .525 dosis de vacuna influenza durante un período del año en el que los países del Hemisferio Sur suelen haber sellado sus adquisiciones de vacuna mientras es el turno de los países del Hemisferio Norte de preparar sus campañas de vacunación. Además, el 2020 se observó un aumento internacional de la demanda de vacuna influenza por el interés de prevenir la concomitancia de influenza con COVID-19 ${ }^{17}$. Estas condiciones, junto con las limitaciones de transporte aéreo que obedecían a la situación sanitaria internacional, impusieron un gran desafío al DI para cumplir oportunamente con la satisfacción del beneficio de vacunación gratuita a los nuevos grupos. Sin embargo, el DI maximizó su capacidad de gestión de compra y en menos de dos meses logró asegurar las dosis de vacuna influenza para los nuevos grupos y, así, garantizó el acceso a vacunación gratuita a toda la población considerada como en mayor riesgo de cursar un cuadro de enfermedad grave por influenza o por concomitancia con COVID-19.

La ejecución de la Campaña Influenza 2020 cursó sin interrupciones, con un descenso de la tasa de notificación de ESAVI respecto de años anteriores y registrando, incluso, una cifra histórica de vacunación diaria, 799.964 dosis el segundo día hábil de implementación. La alta demanda de vacunación hizo que la campaña Influenza adquiriera una velocidad de avance extraordinaria, entre

*Estimación realizada a partir de datos obtenidos del Instituto de Salud Pública por medio de la consulta 21-049. una y dos semanas delante de lo habitual en alcanzar el máximo de dosis administradas al día y cumpliendo con la meta de cobertura de vacunación más de un mes antes de lo observado en otros años.

Junto con la capacidad del DI de reorganizar una campaña de vacunación masiva en curso, la rápida respuesta, apoyo y adaptabilidad a la nueva situación de la campaña por parte de los SEREMI, Servicios de Salud a nivel medio y centros de atención primaria de salud a nivel ejecutor fueron cruciales para su éxito, junto con mantener un alto desempeño técnico. Sin embargo, el alto rendimiento de los actores debe entenderse como un esfuerzo frente a una situación excepcional y no como un escenario ideal, puesto que, para alcanzar un rendimiento de alrededor de 800.000 dosis de vacuna administradas al día, el sistema de entrega del servicio de vacunación ciertamente fue sometido a una exigencia y presión excepcionales. En años previos, libres de pandemia por SARS-CoV-2, el máximo de dosis administradas en un día alcanzaría alrededor de 230.000. Si el año 2020 el manejo comunicacional de la Campaña Influenza contribuyó a la prevención de la concurrencia de influenza y COVID-19, además que debió servir para la difusión de un mensaje de calma y orden para acudir a los puntos de vacunación, el 2021 ésta enfrenta un desafío mayor que es la co-existencia con la vacunación masiva contra SARS-CoV-2. Junto con favorecer el orden, la calma, la prevención de infección y diseminación de SARS-CoV-2, la comunicación en torno a las campañas de vacunación debe dedicar esfuerzos a señalar con claridad la distinta utilidad de cada vacuna, por ejemplo, que la vacuna influenza es efectiva sólo contra la influenza estacional y que las vacunas COVID-19 autorizadas para uso en Chile han demostrado eficacia contra COVID-19. Como bien superior, además, la comunicación sobre vacunación poblacional debe procurar mantener la confianza que la población ha depositado en la vacunación que diseña e implementa el $\mathrm{DI}^{18}$.

En agosto de 2020, el DI comenzó un nuevo ciclo de planificación de la Campaña Influenza, en este caso para el 2021. En diciembre de 2020, el DI ya cerraba la compra de 8,4 millones de dosis de vacuna influenza y había procurado los recursos para la dotación de cada SEREMI con equipo vacunador y tres adicionales en la Región Metropolitana, junto con una encargada de apoyo a cada SEREMI, personal adicional en los depósitos de vacunas e inmunoglobulinas y otros refuerzos que le corresponden a la campaña ${ }^{19}$. Posiblemente, la Campaña Influenza 2021 mostrará un comportamiento distinto a lo observado el 2020 dadas las medidas de prevención y control de SARS-CoV-2 aún impuestas, a la vacunación masiva contra SARS-CoV-2, que consiste en esquemas de dos dosis (primer intervalo de tiempo) que deben separarse por 14 días de otro esquema de vacunación 
(segundo intervalo de tiempo) y que, por tanto, podría extender la ejecución de la Campaña Influenza y desacelerar su velocidad de avance, entre otros factores que revelarán información una vez que la campaña esté en desarrollo. Queda por observar el comportamiento de la notificación de ESAVI en consideración de la disminución que experimentó el 2020. Hasta ese año, las campañas de vacunación contra influenza serían las más grandes implementadas en el país; sin embargo, el 2021 concederán ese título a la vacunación contra
SARS-CoV-2. Sólo el tiempo dirá si la vacunación contra SARS-CoV-2 se mantendrá como la campaña más masiva de Chile (duración de la respuesta inmune, capacidad para interrumpir transmisión viral, autorización de uso en otros grupos de edad y grupos especiales) o si devolverá a la Campaña Influenza su reinado.

Manuscrito revisado por Sylvia Santander Rigollet, jefa de la División de Prevención y Control de Enfermedades, Subsecretaría de Salud Pública, Ministerio de Salud de Chile.

\section{Referencias bibliográficas}

1.- Ministerio de Salud- Gobierno de Chile. Misión del Departamento de Inmunizaciones [Internet]. Available from: https://vacunas.minsal.cl/ conozcanos/mision

2.- Ministerio de Salud de Chile. Plan nacional de preparación para una pandemia de Influenza [Internet]. 2007. Available from: https://www. paho.org/hq/dmdocuments/2010/NIPP_Chile. pdf

3.- Subsecretaría de Salud Pública de Chile. Decreto Ex. No 12 de 09-03-2020. Dispone vacunación obligatoria contra influenza para el año 2020 a grupo de población que indica [Internet]. 2020. Available from: https://www. minsal.cl/wp-content/uploads/2020/03/Decretode-Vacunacion-contra-la-Influenza-2020Diario-Oficial.pdf

4.- World Health Organization. Novel Coronavirs (2019-nCoV) [Internet]. Situation Report 11. 2020 [cited 2020 May 20]. Available from: https://www.who.int/docs/default-source/ coronaviruse/situation-reports/20200131-sitrep11-ncov.pdf?sfvrsn=de 7c0f7 4

5.- World Health Organization. Coronavirus disease 2019 (COVID-19) [Internet]. Situation Report 51. 2020 [cited 2020 May 20]. Available from: https://www.who.int/docs/default-source/ coronaviruse/situation-reports/20200311-sitrep51-covid-19.pdf?sfvrsn=1ba62e57 10

6.- Ministerio de Salud de Chile. Decreto No 4 del 5 enero 2020. Alerta sanitaria por el período que se señala y otorga facultades extraordinarias que indica por emergencia de salud pública de importancia (ESPII) por brote del nuevo coronavirus (2019-NCOV) [Internet]. Chile; 2020 p. 1-7. Available from: https://www.minsal.cl/wp-content/ uploads/2020/02/1724518_alerta_sanitaria_ coronavirus.pdf

7.- $\quad$ Ministerio de Salud de Chile. Decreto $N^{\circ} 6$ del 6 de marzo 2020. Modifica decreto $\mathrm{n}^{\circ} 4$ de 2020 del Ministerio de Salud, que decreta alerta sanitaria por el período que se señala y otorga facultades extraordinarias que indica por emergencia de salud pública de importancia internacional [Internet]. № 10 del 24 marzo 2020 Chile; 2020. Available from: https://cdn. digital.gob.cl/filer_public/29/d2/29d21b1a21df-4cf0-9554-f3e5d366d342/do_1_1745010. pdf

8.- MINSAL. Autoridades lanzan camapaña de vacunación contra la Influenza [Internet]. 2020 [cited 2021 Jan 27]. Available from: https:// www.minsal.cl/autoridades-lanzan-campanade-vacunacion-contra-la-influenza/

9.- Departamento de Inmunizaciones- Ministerio de Salud de Chile. Lineamientos Técnicos Operativos Vacuna Antiinfluenza 2020 [Internet]. 2020. Available from: https:// vacunas.minsal.cl/wp-content/uploads/2020/03/ Lineamientos-Tecnico-Operativos-CampañaInfluenza-2020.pdf

10.- González C, Cofré J. Recomendaciones para la vacunación de pacientes con necesidades especiales por patologías o situaciones de riesgo [Internet]. 2018. Available from: https:// vacunas.minsal.cl/wp-content/uploads/2019/02/ MANUAL-VACUNAS-ESPECIALES.pdf

11.- World Health Organization. Recommended composition of influenza virus vaccines for use in the 2020 southern hemisphere influenza season [Internet]. 27 September 2019. [cited 2021 Jan 27]. Available from: https://www.who.int/influenza/vaccines/virus/ recommendations/2020 south/en/

12.- Departamento de Inmunizaciones- Ministerio de Salud de Chile. Guía de Vacunación Segura [Internet]. 2013. Available from: https:// vacunas.minsal.cl/wp-content/uploads/2015/06/ norma- $\mathrm{N}^{\circ} 670$-Guía-Vacunacion-Segura-8oct-2013.pdf

13.- Subsecretaría de Salud Pública-Ministerio de Salud de Chile. Ordinario B27 No 136029 abril 2020 Recomendaciones para la vacunación programática durante pandemia COVID-19 [Internet]. 2020. Available from: https:// vacunas.minsal.cl/wp-content/uploads/2020/07/ Ord-B27-N ${ }^{\circ}-1360$-Recomendaciones-paravacunacion-programatica-durante-pandemiacovid19-29-abril-2020.pdf\%0A\%0A
14.- Subsecretaría de Salud Pública-Ministerio de Salud de Chile. Ordinario B27 № 1389 del 6 de mayo 2020 Recomendaciones para la vacunación escolar extramural durante la pandemia COVID-19 [Internet]. 2020. Available from: https://vacunas.minsal.cl/ wp-content/uploads/2020/07/Ord-B27-N ${ }^{\circ}$ 1389-Recomendaciones-para-la-vacunacionescolar-extramular-durante-pandemia-covid196-mayo-2020.pdf

15.- Subsecretaría de Salud Pública-Ministerio de Salud de Chile. Ordinario B27 №2148 12 junio 2020 Recomendaciones para la vacunación de recién nacidos durante la pandemia COVID-19 [Internet]. 2020. Available from: https://vacunas.minsal.cl/ wp-content/uploads/2020/07/Ord-B27-N ${ }^{\circ}$ 2148-Recomendaciones-para-la-vacunacion-derecien-nacidos-durante-la-pandemia-covid1912-junio-2020.pdf

16.- Subsecretaría de Salud Pública-Ministerio de Salud de Chile. Ordinario B27 № 3021 del 3 de agosto 2020 Recomendaciones para cierre de campaña de vacunación contra Influenza durante pandemia COVID-19 [Internet]. 2020 Available from: https://vacunas.minsal.cl/wpcontent/uploads/2020/08/Ord-B27-N ${ }^{\circ}-3021$ Recomendaciones-para-cierre-de-campañade-vacunación-contra-la-Influenza-durantepandemia-Covid-19-03-agosto-2020.pdf

17.- UNICEF. Seasonal Influenza Vaccine: Supply Update [Internet]. 2020. Available from: https://www.unicef.org/supply/media/5206/ file/Seasonal-influenza-vaccine-supply-noteSeptember-2020.pdf

18.- CAVEI. Recomendación del CAVEI sobre contenidos a considerar en una campaña comunicacional de vacunación COVID-19 [Internet]. 2020. Available from: https:// vacunas.minsal.cl/wp-content/uploads/2020/12/ Recomendación-CAVEI-contenidos-esencialescomunicación-COVID19_16Dic2020.pdf

19.- CAVEI. Acta sesión ordinaria CAVEI 28 de octubre 2020 [Internet]. Available from: https:// vacunas.minsal.cl/wp-content/uploads/2020/11/ ACTA_CAVEI_28_OCT2020_final.pdf 